rheuma plus $2018 \cdot 17: 1$

https://doi.org/10.1007/s12688-018-0171-5

(c) Springer-Verlag GmbH Austria, ein Teil von

Springer Nature 2018

\section{Burkhard Leeb}

II. Medizinische Abteilung, NOE Kompetenzzentrum für Rheumatologie, Stockerau, Österreich

\title{
Wird 2018 ein Jahr der Veränderungen?
}

\section{Liebe Leserinnen und Leser!}

2018 werden wieder Veränderungen auf uns zukommen. Die wohl brennendste Frage: Wird es zu substanziellen Veränderungen im Sozialversicherungswesen kommen? Ein funktionierendes Chefarztsystem hätte wohl den Analgetika-Missbrauch (5000 Tabletten verschrieben in ganz Österreich für eine Person in einem halben Jahr) aufdecken können müssen. Wird es notwendige Veränderungen der Krankenhauslandschaft geben? Der Krankenhausbetrieb birgt viel Veränderungspotential zum Positiven und auch Einsparungspotential. Denn zu viele „Experten“ glauben, man könne das Gesundheitswesen organisieren wie eine Schraubenfabrik mit Meetings, Zielvereinbarungen, Regulierungen und standardised operating procedures (SOPs), was entsprechend negative Auswirkungen zeitigt und Ressourcen vernichtet.

Selbstverständlich wird es auch an uns Rheumatologen liegen auf die geänderte Situation, vor allem was die Arbeitszeit und die Verfügbarkeit von Ärzten betrifft, zu reagieren. Kontrollmechanismen, die nicht unbedingt die Face To Face-Interaktion von Patient und Arzt bedingen, werden eine größere Rolle spielen müssen. Webbasierte Lösungen unter Zuhilfenahme von Patient Related Outcomes, werden in verstärktem Maße zum Monitoring eingesetzt werden müssen mit dem Vorteil, dem Betroffenen eine bedeutend stärkere Rolle im Krankheitsmanagement zuzuweisen. Damit wird auch die Lawine an teilweise fraglich wertvollen Empfehlungen neu zu bewerten sein, denn es wird dann schwerer werden, Standardisierungsmanien zu frönen. Die Österreichische Gesellschaft für Innere Medizin hat ja mit dem Generalthema des Kongresses 2017 „Empfehlungen versus Erfahrungsmedizin“ in dieser Richtung ein Zeichen gesetzt und verstärkt dieses mit dem Generalthema „Personalisierte Medizin“ im Jahre 2018. Die Rheumatologie wäre gut beraten, diesem Beispiel zu folgen und nicht das in durchaus redlicher Weise zugegebene Nicht-bzw. Halbwissen in eine Flut von Empfehlungen zu verpacken.

Eine erfreuliche Veränderung, die rheuma plus betrifft: Sie, liebe Leserinnen und Leser, haben unserem Journal so viel Vertrauen geschenkt, dass man sich seitens des Verlages entschlossen hat, die Zahl der jährlichen Ausgaben auf sechs zu erhöhen. Das gesamte Team von rheuma plus freut sich aufrichtig über diesen Vertrauensvorschuss und wird sich bemühen diesem auch gerecht zu werden. Neue bzw. vertiefte Schwerpunkte werden Fallberichte aus der Praxis bzw. auch verstärkte Informationen über BioReg, das österreichische Biologika-Register darstellen.

In dieser Ausgabe von rheuma plus wird dem Schwerpunkt Fallbeispiele durch Ulrike Stummers (Stockerau) Beitrag über einen Patienten mit einer nicht ganz alltäglichen Oligoarthritis Rechnung getragen. Infektiöse Arthritiden sind zweifellos Raritäten, aber stellen doch eine ganz wesentliche Differenzialdiagnose bei der Abklärung entzündlicher Gelenkerkrankungen dar. Dem zweiten geplanten zukünftigen Fokus entspricht die Zusammenfassung des Datenreports 2017 über Patienten mit rheumatoider Arthritis aus dem österreichischen Biologikaregister BioReg, in das mittlerweile mehr als 2100 Patienten eingeschlossen wurden.

Elisabeth Preisinger (Hietzing) streicht die Bedeutung des körperlichen Trainings für eine erfolgreiche Behandlung der Osteoporose hervor - mit dem Succus, dass eine Unterforderung der Patienten bei körperlichen Aktivitäten nicht zielführend sei. Auch die Kinderrheumatologie kommt in diesem Heft zu Wort. Die Kawasaki-Erkrankung stellt eine der häufigeren Vaskultitiden im Kindesalter dar. Ulrich Neudorf (Essen) gibt einen Überblick über Pathogenese, Diagnostik und Therapie.

Auch mit dieser Ausgabe hoffen wir, wieder Ihr Interesse zu wecken und dem Ziel von rheuma plus nahe zu kommen, eine fruchtbringende, offene und redliche Diskussion in Gang zu bringen, aus der wir alle Nutzen ziehen können. Wie immer an dieser Stelle möchten wir Sie herzlich dazu einladen, uns Ihre Meinung zu den Beiträgen dieser Ausgabe, wie auch zu allen rheumatologischen Themen, die Sie für relevant oder für diskussionswert halten, zu schicken. Sie können in jedem Falle auf einen fairen Review mit entsprechendem Feedback vertrauen. Für Kritik, Hinweise, Zustimmung wie auch für jeden anderen Beitrag bedanke ich mich schon im Voraus.

\section{Herzlichst,}

Ihr Burkhard Leeb

\section{Korrespondenzadresse}

Burkhard Leeb
II. Medizinische Abteilung,
NOE Kompetenzzentrum für
Rheumatologie
Stockerau, Österreich
leeb.rheuma@aon.at

